\title{
Diagnostic value of a hand-carried ultrasound device for free intra-abdominal fluid and organ lacerations in major trauma patients
}

\author{
Stephan Schleder, ${ }^{1}$ Lena-Marie Dendl, ${ }^{1}$ Antonio Ernstberger, ${ }^{2}$ Michael Nerlich, ${ }^{2}$ \\ Patrick Hoffstetter, ${ }^{1}$ Ernst-Michael Jung, ${ }^{1}$ Peter Heiss, ${ }^{1}$ Christian Stroszczynski, ${ }^{1}$ \\ Andreas G Schreyer ${ }^{1}$
}

${ }^{1}$ Department of Radiology, University Medical Center Regensburg, Regensburg, Germany

${ }^{2}$ Department of Trauma Surgery, University Medical Center Regensburg,

Regensburg, Germany

Correspondence to Dr Stephan Schleder, Department of Radiology, University Medical Center Regensburg,

Franz-Josef-Strauss-Allee 11, Regensburg 93053, Germany: stephan.schleder@ukr.de

Accepted 15 March 2012 Published Online First 19 April 2012

\begin{abstract}
Background Technological progress has led to the introduction of hand-carried ultrasound (HCU) imagers in clinical workflow. The aim of this study is to analyse whether examination with a HCU device is a rapid and reliable alternative to contrast-enhanced multidetector CT (MDCT) scans in diagnosis of free intra-abdominal fluid and organ lacerations in major trauma patients.

Methods 31 major trauma patients with an injury severity score $>15$ and the necessity of a MDCT scan (standard of reference) were enrolled prospectively to this study, and additionally examined with a HCU, according to 'focused assessment with sonography for trauma' principles for the assessment of organ lacerations and free intra-abdominal fluid. The HCU device employed was of the latest generation. Statistical analysis was performed using PASW V.18.

Results Four patients were diagnosed with free intraabdominal fluid (prevalence 12.9\%). HCU showed a sensitivity and specificity of $75 \%$ and $100 \%$, respectively. Positive predictive value and negative predictive value were $100 \%$ and $96 \%$, respectively. Five patients had organ lacerations (prevalence 16.1\%). In these cases, the HCU was able to detect organ lacerations with a sensitivity and specificity of $80 \%$ and $100 \%$, respectively. Therefore, a positive predictive value and negative predictive value of $100 \%$ and $96 \%$, respectively, were calculated.
\end{abstract}

Conclusion In major trauma patients, examination with $\mathrm{HCU}$ according to the 'focused assessment with sonography for trauma' principles for the diagnosis of organ lacerations and free intra-abdominal fluid is a reliable and rapid alternative to MDCT scans and can help save precious time in emergency situations, and should, additionally, be evaluated in the pre-clinical workflow.

\section{INTRODUCTION}

Over the past few years, the frequency of intraabdominal injuries from blunt abdominal trauma continues to increase worldwide. ${ }^{1}$ This entity is life-threatening and a major cause of death within the first $24 \mathrm{~h}$ after trauma, requiring immediate action by all physicians involved. ${ }^{2}{ }^{3}$ The organs being most frequently involved are the spleen and liver. $^{4} 5$

Abdominal injuries have historically been difficult to detect, and a delayed diagnosis increases the length of hospitalisation, morbidity and mortality. ${ }^{67}$ Diagnostic peritoneal lavage has a high sensitivity for the detection of abdominal injuries, but as an invasive procedure, it is associated with complications and, consequently, disadvantageous for haemodynamically unstable trauma patients. ${ }^{89}$ $\mathrm{CT}$ is considered as a sensitive testing method for blunt abdominal trauma. Especially, the multidetector CT (MDCT) represents state-of-the-art technology for major trauma patients. Still, this modality is not available $24 \mathrm{~h}$ and 7 days a week, and even less so in rural regions. Moreover, it is considered to be time-consuming and expensive, as the patient needs transportation and trained personnel are required. ${ }^{10}$

However, ultrasound provides a quick and standardised overview of the abdomen with a high sensitivity for free intra-abdominal fluid collections, but cannot distinguish between blood, urine, ascites or bile. ${ }^{71}$ However, it is an excellent screening method. In 1997, the International Consensus Conference Committee defined the acronym 'FAST', which stands for 'focused assessment with sonography for trauma'. Since the late 1990s, it has been the clinical standard for the identification of free intra-abdominal fluid accumulating either in Morison's pouch, Coller's pouch or the pouch of Douglas in major trauma patients after blunt abdominal trauma. ${ }^{12}$

With further developments in the field of miniaturised ultrasound systems, hand-carried ultrasound (HCU) imagers have also been introduced into the everyday clinical workflow. The major advantage of these devices is that it is a fast bedside instrument, pre-hospital available and is excellent in transportability. All this leads to a prompt diagnosis and the possibility of immediate therapeutic measures at low costs. Prior studies by Brooks et al, Kirkpatrick et al and Walcher et al have already shown promising results with HCU devices in the clinical and pre-clinical routine allowing appropriate triage of patients. ${ }^{13-16}$ However, all these studies have been conducted in special settings, for example, military or air medical environments. ${ }^{13} 15$ The HCU devices used were, for example, Sonosite 180 (Sonosite, Bothell, Washington, USA) or Premedic Handyscan (Metrax, Rottweil, Baden-Württemberg, Germany) with a weight ranging from $800 \mathrm{~g}$ to $2400 \mathrm{~g}$.

The wide deployment of a HCU device, reliability in diagnosis and ease in transportability, could speed up the pre-hospital diagnosis of blunt 
abdominal trauma and help in saving time for a patient's triage and selection of the appropriate trauma centre in coping with urgent situations.

In this prospective study, we evaluated the diagnostic yield of a new-generation HCU imager in comparison with a contrastenhanced MDCT scan as standard of reference in patients with major trauma concerning the diagnosis of free intra-abdominal fluid or organ lacerations.

\section{METHODS}

\section{Patients}

From December 2010 to March 2011, a total of 64 patients were admitted to the emergency department unit diagnosed with major trauma, based on an injury severity score $>15,{ }^{17}$ and all of them underwent a contrast-enhanced MDCT scan. Only patients admitted to the emergency department within the core service hours of the Department of Radiology, that is, 8:00 to 17:00 on working days were included in the study to guarantee ultrasound examination by only one examiner. This leads to a total of 31 patients being additionally examined with a HCU for the presence or absence of free intra-abdominal fluid and organ lacerations according to the 'FAST' principles. ${ }^{12}$ The patients' basic demographic data are shown in table 1.

\section{Ethics}

The study was approved by the ethics committee of the Medical Faculty of the University Medical Centre Regensburg. As only major trauma patients with an injury severity score $>15$ were included, only verbal informed consent could be obtained, which was specifically approved by the ethics committee.

\section{Imaging}

The contrast-enhanced MDCT scans were acquired using either a SOMATOM Sensation 16 (Siemens, Erlangen, Bayern, Germany) or a SOMATOM Definition Flash (Siemens). All patients examined with SOMATOM Sensation 16 (16 slices CT) were given $150 \mathrm{ml}$ Accupaque 300 intravenously (GE Healthcare, Waukesha, Wisconsin, USA). If diagnosis was made using SOMATOM Definition Flash (128 slices CT), $120 \mathrm{ml}$ Ultravist 370 (Bayer Vital, Leverkusen Nordrhein-Westfalen, Germany) was injected intravenously. In both cases, the decision on the quantity of the contrast agent was based on the standardised major trauma examination protocol. The MDCT scan was evaluated for the presence or absence of free intra-abdominal fluid, or organ lacerations, by the same radiologist (AGS), who has expertise of over 5 years in abdominal imaging, and who was blinded to the ultrasonographic and clinical findings.

For the ultrasound examination, a new-generation HCU device (VScan, GE Healthcare) with a plane $1.7-3.8 \mathrm{MHz}$ transducer for two-dimensional imaging was employed (figure 1). The 3.5-inch colour LCD display measured $135 \times 73 \times 28 \mathrm{~mm}$, whereas the probe measured $120 \times 33 \times 26 \mathrm{~mm}$.

Table 1 Basic demographic characteristics of enrolled patients

\begin{tabular}{lll}
\hline & Absolute numbers & Percentage (\%) \\
\hline Patients & 31 & 100 \\
Gender & & \\
$\quad$ Male & 19 & 61 \\
$\quad$ Female & 12 & 39 \\
Median age and age range (years) & $50(18-80)$ & \\
Median weight and weight & $81(58-96)$ & \\
range (kilograms) & & \\
\hline
\end{tabular}

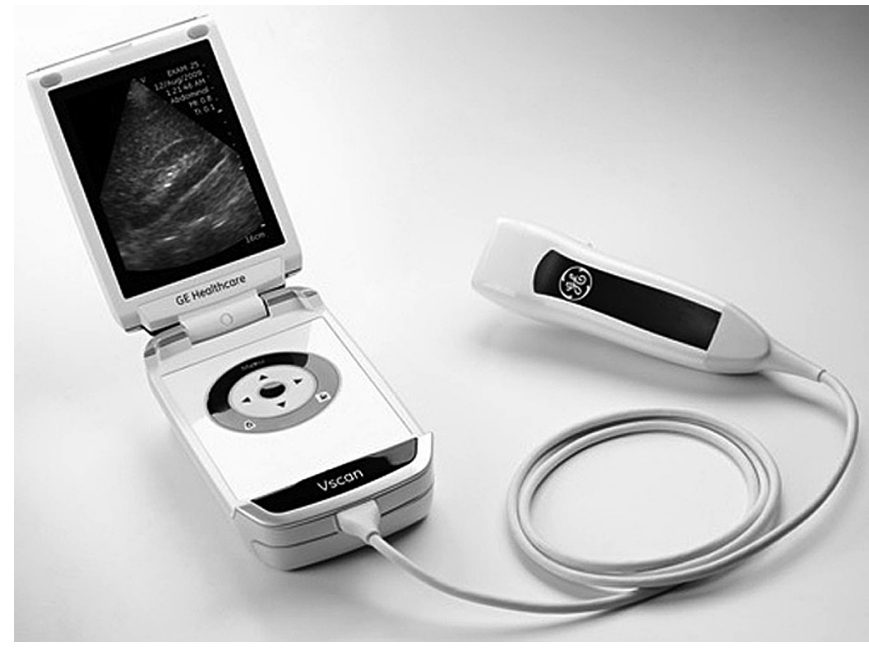

Figure 1 Image of the hand-carried ultrasound device (VScan, GE Healthcare, Waukesha, Wisconsin, USA) - with kind permission of $\mathrm{Mr}$ Bastian Werminghoff (GE Healthcare, Munich, Bayern, Germany).

The weight of the device was $390 \mathrm{~g}$, and the total scan time with fully charged batteries was about $62 \mathrm{~min}$. The ultrasound examination following the 'FAST' approach with the HCU imager was performed on the CT table right before the acquisition of the contrast-enhanced MDCT scan. The operator (SS) was a radiologist with a practice of more than 1000 documented ultrasound examinations in the past 12 months, and under the supervision of an experienced ultrasound examiner who has been conducting more than 5000 ultrasound examinations each year for more than 10 years. The operator was also unaware of the contrast-enhanced MDCT scan results or the clinical findings.

Following the 'FAST' guidelines ${ }^{12}$ for the abdomen, Morison's pouch, Coller's pouch and the pouch of Douglas were songraphically scanned followed by a short examination of the pericardial sac, and evaluated for free intra-abdominal fluid and organ lacerations.

\section{Statistical analysis}

Data was documented using Excel tables (Excel 2007, Microsoft, Redmond, Washington, USA). Statistical analysis was performed using PASW V.18 (PASW V.18, IBM SPSS Inc., Armonk, New York, USA). Agreement between the findings of HCU with the diagnosis made by contrast-enhanced MDCT scan as standard of reference was assessed from $2 \times 2$ tables.

\section{RESULTS}

HCU was carried out successful in all 31 patients with a mean scanning time of approximately $60 \mathrm{~s}$ ranging from $45 \mathrm{~s}$ to $1.5 \mathrm{~min}$.

The reading of the contrast-enhanced MDCT scans diagnosed free intra-abdominal fluid in four out of 31 patients resulting in a prevalence of $12.9 \%$ patients with free intra-abdominal fluid.

$\mathrm{HCU}$ examination revealed free intra-abdominal fluid correctly in three out of four patients with no false positive results. The absence of free intra-abdominal fluid was correctly verified by HCU in all 27 patients. Based on our data, this results in a sensitivity of $75 \%$ and a specificity of $100 \%$. In the single case where the HCU examination was false negative, analysis of the contrast-enhanced MDCT showed approximately $20 \mathrm{ml}$ fluid in the pouch of Douglas (figure 2). Positive predictive value (PPV) and negative predictive value (NPV) for the diagnosis of 
Figure 2 In a 75-year-old man, a fracture of the left acetabulum and a surrounding haematoma, additionally, with small amounts of free intraabdominal fluid (approximately $20 \mathrm{ml}$ ) following a car accident, were found using contrast-enhanced multidetector CT. There was no evidence of free intraabdominal fluid collection in the handcarried ultrasound examination. (A) Longitudinal B-Scan hand-carried ultrasound image of the urinary bladder giving no evidence of the small amount of free intra-abdominal fluid. (B) Contrast-enhanced multidetector CT scan image showing haematoma beneath the left acetabulum (marked with white arrow) and small amounts of free intra-abdominal fluid (marked with black arrow).
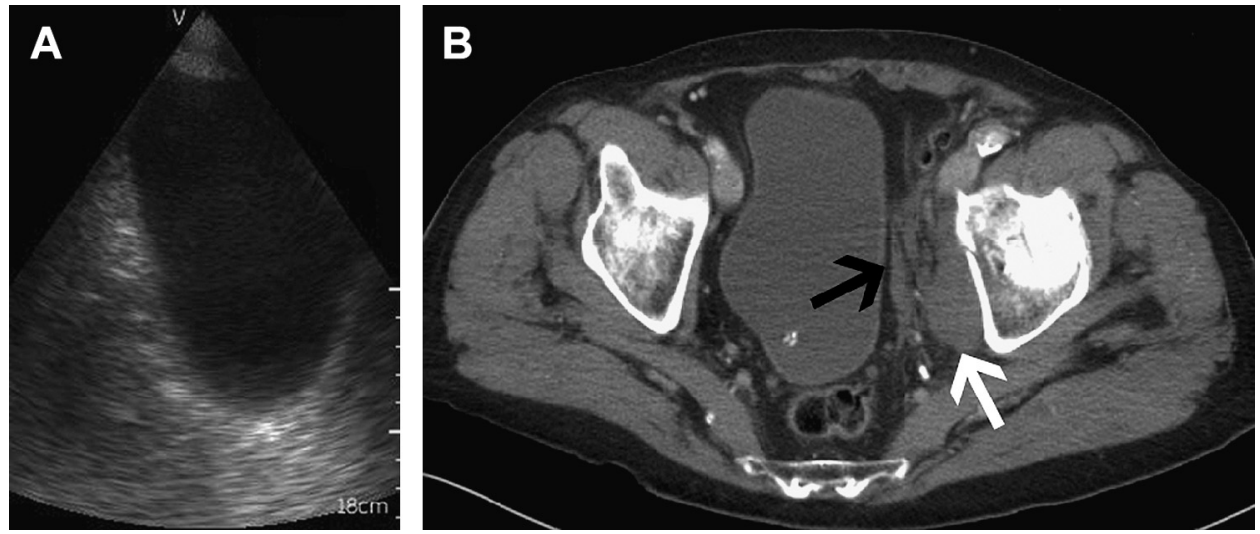

free intra-abdominal fluid collection with HCU were $100 \%$ and $96 \%$, respectively.

The statistics of diagnosis and assessment of free intraabdominal fluid by means of HCU are summarised in table 2 .

The contrast-enhanced MDCT scan depicted organ lacerations in five out of 31 patients resulting in a prevalence of $16.1 \%$ with organ lacerations. In four patients, a liver laceration was detected, and in another patient, a mesenteric bleeding was found. In all 31 patients, no injury of the spleen could be found.

Examination with a HCU revealed all four liver lacerations correctly and showed no false positive results. The mesenteric bleeding was not recognised correctly by the HCU, which resulted in one false negative case. A sensitivity of $80 \%$ with a specificity of $100 \%$ for the diagnosis of organ lacerations was calculated. PPV and NPV for the diagnosis of organ lacerations with the HCU were $100 \%$ and $96 \%$, respectively.

The HCU results of organ lacerations are summarised in table 3. An example of one of the patients with a liver laceration diagnosed in both HCU and MDCT examination is shown in figure 3 .

\section{DISCUSSION}

Abdominal ultrasound is a common and accepted method for the evaluation of intra-abdominal injuries in major trauma patients following blunt abdominal trauma. ${ }^{18}$ Its sensitivity and specificity for the exclusion of free intra-abdominal fluid using conventional ultrasound B-Scan have been reported by several authors, with $81-89 \%$ and $93-100 \%$, respectively. ${ }^{19-21}$ These numbers are supported by the data acquired in our study.

Diagnosis of organ lacerations based on ultrasound examinations proved more challenging. Lacerations of the liver or spleen are better evaluated, while data concerning pancreas, gallbladder or bile duct injuries are quite rare. ${ }^{4} 520$
HCU imagers have been tested previously in the assessment of major trauma patients concerning abdominal injuries, and these prior studies have also been disclosing promising findings, but HCU imagers have not yet made their way into clinical daily workflow. ${ }^{1622-24}$ HCU devices have even been employed in prehospital settings. Walcher et al and Ruesseler et al demonstrated their reliability and usefulness earlier. ${ }^{16} 2122$

In 2002, Walcher et al conducted a study with 61 abdominal trauma patients who were examined using a Premedic Handyscan (Metrax), following 'FAST' guidelines, ${ }^{12}$ directly at the accident scene. After admission routine, an ultrasound and MDCT scan were obtained to control the pre-hospital ultrasonographic findings. The mean investigation time was $2.8 \mathrm{~min}$, and in 16 patients, free intra-abdominal fluid was detected (26.2\%). There was one false positive and no false negative result in HCU examinations for the diagnosis of intra-abdominal fluid and, therefore, a sensitivity of $97.9 \%$ and a specificity of $100 \%$ were calculated leading to a PPV of $94.2 \%$ and an NPV of $100 \%{ }^{16}$

In 2004, Kirkpatrick et al tested a HCU imager in patients with penetrating abdominal trauma. A total of 38 patients were examined with the Sonosite 180 (Sonosite) in a clinical environment according to the 'FAST' guidelines. ${ }^{12}$ Definite diagnosis of free intra-abdominal fluid was made with a routine ultrasound scan with the Acuson XP128 (Acuson, Mountain View, California, USA) or the Toshiba SSH 140A (Toshiba American Medical Systems, Tustin, California, USA). If available, MDCT results, operative findings and autopsy studies were considered. Free intra-abdominal fluid was detected correctly in 11 of 12 patients by means of $\mathrm{HCU}$, and there was only one false negative case. Therefore, a sensitivity of $91.7 \%$ and a specificity of $100 \%$ were calculated, and a PPV and an NPV of $100 \%$ and $96.3 \%$, respectively, were found for the diagnosis of free intra-abdominal fluid. ${ }^{23}$
Table 2 Diagnosis of free intra-abdominal fluid using the hand-carried ultrasound device with a contrast-enhanced multi-detector CT scan as standard of reference

\begin{tabular}{lc}
\hline & $\begin{array}{c}\text { Diagnosis of free } \\
\text { intra-abdominal fluid (\%) }\end{array}$ \\
\hline Sensitivity & 75 \\
Specificity & 100 \\
Positive predictive value & 100 \\
Negative predictive value & 96 \\
\hline
\end{tabular}

Table 3 Diagnosis of organ lacerations using hand-carried ultrasound imager with a contrast-enhanced multi-detector CT scan as standard of reference

\begin{tabular}{lc}
\hline & $\begin{array}{c}\text { Diagnosis of organ } \\
\text { lacerations (\%) }\end{array}$ \\
\hline Sensitivity & 80 \\
Specificity & 100 \\
Positive predictive value & 100 \\
Negative predictive value & 96 \\
\hline
\end{tabular}


Figure 3 In a 63-year-old woman, a sub-capsular liver haematoma and a hepatic laceration were congruently diagnosed by contrast-enhanced multidetector CT scan and hand-carried ultrasound after she fell down some stairs. (A) Transverse B-Scan handcarried ultrasound image of the liver showing the sub-capsular liver haematoma (marked with white arrow), and the hepatic laceration (marked with black arrow). (B) Contrast-enhanced multidetector CT scan image, showing sub-capsular liver haematoma (marked with white arrow) and the liver laceration (marked with black arrow).
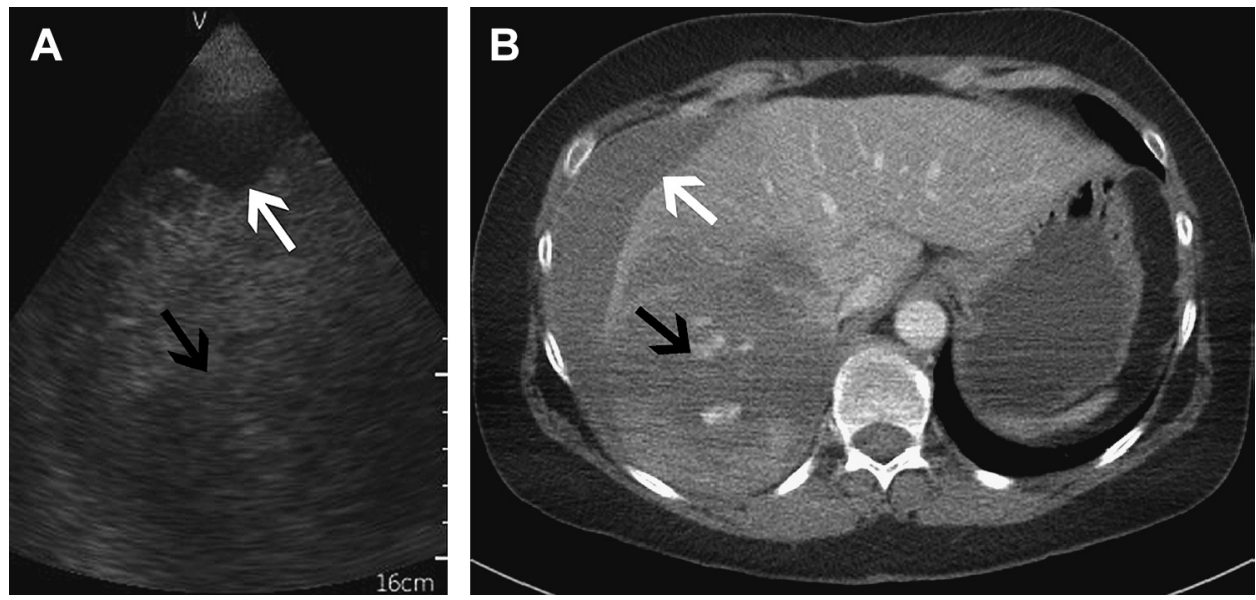

However, the results of prior studies, and the results of this current study, indicate that modern $\mathrm{HCU}$ imagers provide an excellent alternative for the detection of free intra-abdominal fluid collections and organ lacerations in comparison with contrast-enhanced MDCT scans. Absence of radiation and quick availability, and low costs and mobility, are additional advantages. As mentioned above, this study was performed with a latest-generation HCU device (VScan, GE Healthcare) with a plane $1.7-3.8 \mathrm{MHz}$ transducer for two-dimensional imaging, and a 3.5-inch colour LCD-display with a weight of only $390 \mathrm{~g}$, and a scanning time of approximately $1 \mathrm{~h}$ with a set of fully charged batteries. Such modern devices are easy and quick to handle and are highly recommended by the authors for use in emergency situations like major trauma patients or blunt abdominal traumas in general.

Based on our results, comparing a HCU examination to a state-of-the-art MDCT scan, and with the ultrasound scan being conducted directly on the CT table and immediately prior to the CT scan, we consider the quality of a HCU examination in all cases sufficient for a successful screening for free intraabdominal fluid and organ lacerations in patients with major trauma. The use of such small and light HCU devices in a preclinical trauma setting could be helpful, especially for triage and fast patient transport to an appropriate trauma centre. But, however, the authors are convinced that HCU imagers cannot replace the use of either the high-end ultrasound imagers because of the better image quality, or of the MDCT scans because of the better recognition of oblique lesions (like the one case of mesenteric bleeding in this current study) in the near future.

\section{LIMITATIONS}

Limiting factors of the study might be that only 31 patients were included, and the incidence of severe abdominal injury was generally low. Still, patients were prospectively acquired representing the daily routine in a tertiary care emergency centre. Furthermore, only one operator was working on the HCU system, and ultrasound examination is normally an operatordependent modality, with different operators likely to obtain different results. However, we are convinced that only experienced operators are qualified in conducting the examination with HCU imagers, while inexperienced operators might not obtain the correct diagnosis in urgent situations.

\section{CONCLUSION}

The use of a HCU device according to the 'FAST' principles for the examination of major trauma patients is reliable for the diagnosis of free intra-abdominal fluid and organ lacerations, and can help save precious time in emergency situations. The diagnostic advantages of latest-generation $\mathrm{HCU}$ devices for the detection of free intra-abdominal fluid and organ lacerations in a pre-clinical workflow should be evaluated further.

Contributors SS, AE, E-MJ and P Heiss contributed to the conception and design of the study. SS and AGS were involved in analysis and interpretation of data and along with L-MD drafted the manuscript. AE, MN, E-MJ, CS and P Hoffstetter critically revised the manuscript. All the authors read approved the revised manuscript for submission.

\section{Competing interests None.}

Patient consent As only major trauma patients with an injury severity score $>15$ were included, only verbal informed consent could be obtained, and this was specifically approved by the ethics committee.

Ethics approval The ethics approval was provided by the Ethics Committee of the Medical Faculty, University Medical Centre Regensburg, Germany.

Provenance and peer review Not commissioned; internally peer reviewed.

\section{REFERENCES}

1. Richards JR, McGahan JP, Simpson JL, et al. Bowel and mesenteric injury: evaluation with emergency abdominal US. Radiology 1999;211:399-403.

2. Burney RE, Mueller GL, Coon WW, et al. Diagnosis of isolated small bowel injury following blunt abdominal trauma. Ann Emerg Med 1983:12:71-4.

3. Nast-Kolb D, Waydhas C, Kastl S, et al. [The role of an abdominal injury in follow-up of polytrauma patients] (In German). Chirurg 1993;64:552-9.

4. Raptopoulos V. Abdominal trauma. Emphasis on computed tomography. Radiol Clin North Am 1994;32:969-87.

5. Pohlenz 0, Bode PJ. The trauma emergency room: a concept for handling and imaging the polytrauma patient. Eur J Radiol 1996;22:2-6.

6. Richards JR, Derlet RW. Computed tomography and blunt abdominal injury: patient selection based on examination, haematocrit and haematuria. Injury 1997;28:181-5.

7. Rozycki GS, Ballard RB, Feliciano DV, et al. Surgeon-performed ultrasound for the assessment of truncal injuries: lessons learned from 1540 patients. Ann Surg 1998;228:557-67.

8. Root HD, Hauser CW, McKinley CR, et al. Diagnostic peritoneal lavage. Surgery 1965;57:633-7.

9. McKenney M, Lentz K, Nunez D, et al. Can ultrasound replace diagnostic peritoneal lavage in the assessment of blunt trauma? J Trauma 1994;37:439-41.

10. Boulanger BR, McLellan BA. Blunt abdominal trauma. Emerg Med Clin North Am 1996;14:151-71

11. Boulanger BR, McLellan BA, Brenneman FD, et al. Emergent abdominal sonography as a screening test in a new diagnostic algorithm for blunt trauma. J Trauma 1996; 40:867-74.

12. Scalea TM, Rodriguez A, Chiu WC, et al. Focused Assessment with Sonography fo Trauma (FAST): results from an international consensus conference. J Trauma 1999:46:466-72.

13. Brooks AJ, Price V, Simms M. FAST on operational military deployment. Emerg Med J 2005;22:263-5.

14. Kirkpatrick AW, Sirois M, Laupland KB, et al. Prospective evaluation of hand-held focused abdominal sonography for trauma (FAST) in blunt abdominal trauma. Can $J$ Surg 2005;48:453-60. 
15. Kirkpatrick AW, Breeck K, Wong J, et al. The potential of handheld trauma sonography in the air medical transport of the trauma victim. Air Med J 2005;24:34-9.

16. Walcher F, Kortum S, Kirschning T, et al. [Optimized management of polytraumatized patients by prehospital ultrasound] (In German). Unfallchirurg 2002;105:986-94.

17. Baker SP, O'Neill B, Haddon W Jr, et al. The injury severity score: a method for describing patients with multiple injuries and evaluating emergency care. J Trauma 1974;14:187-96.

18. Porter JM, Rotondo MF. Visceral injury. Curr Opin Gen Surg 1993:62-8.

19. Kretschmer K, Bohndorf K, Pohlenz 0 . The role of sonography in abdominal trauma: the European experience. Emerg Radiol 1997;4:62-7.
20. Rothlin MA, Naf R, Amgwerd M, et al. Ultrasound in blunt abdominal and thoracic trauma. J Trauma 1993;34:488-95.

21. Ruesseler M, Kirschning T, Breitkreutz R, et al. Prehospital and emergency Departement ultrasound in blunt abdominal trauma. Eur J Trauma Emerg Surg 2009;4:341-6.

22. Walcher $\mathbf{F}$, Weinlich $\mathbf{M}$, Conrad $\mathrm{G}$, et al. Prehospital ultrasound imaging improves management of abdominal trauma. Br J Surg 2006;93:238-42.

23. Kirkpatrick AW, Sirois M, Ball CG, et al. The hand-held ultrasound examination for penetrating abdominal trauma. Am J Surg 2004;187:660-5.

24. Wherry DC. Potential of a hand-held ultrasound in assessment of the injured patient. Cardiovasc Surg 1998;6:569-72. 
Diagnostic value of a hand-carried ultrasound device for free intra-abdominal fluid and organ lacerations in major trauma patients

Stephan Schleder, Lena-Marie Dendl, Antonio Ernstberger, Michael Nerlich, Patrick Hoffstetter, Ernst-Michael Jung, Peter Heiss, Christian Stroszczynski and Andreas G Schreyer

Emerg Med J 2013 30: e20 originally published online April 19, 2012 doi: 10.1136/emermed-2012-201258

Updated information and services can be found at:

http://emj.bmj.com/content/30/3/e20

References This article cites 23 articles, 1 of which you can access for free at: http://emj.bmj.com/content/30/3/e20\#BIBL

Email alerting Receive free email alerts when new articles cite this article. Sign up in the service box at the top right corner of the online article.

Topic Articles on similar topics can be found in the following collections Collections

\section{Notes}

To request permissions go to:

http://group.bmj.com/group/rights-licensing/permissions

To order reprints go to:

http://journals.bmj.com/cgi/reprintform

To subscribe to BMJ go to:

http://group.bmj.com/subscribe/ 